\title{
Secondary Electron Imaging in an Aberration-Corrected STEM
}

\author{
D. R. G. Mitchell ${ }^{\star}$ and G. Casillas
}

Electron Microscopy Centre, Australian Institute for Innovative Materials, University of Wollongong, NSW 2500, Australia

*dmitchel@uow.edu.au

Abstract: The aberration-corrected JEOL ARM200F, equipped with a multichannel plate detector, enables routine secondary electron (SE) and backscattered electron (BSE) imaging in STEM mode. Atomic resolution in SE mode is demonstrated, but the real benefits arise at lower magnifications. Depth information is largely absent in brightfield and HAADF STEM projection images. Imaging with SEs allows identification of phases lying on the upper surface of the support film, which is useful knowledge in light element X-ray microanalysis. Topographic information provided by the SE signals improves interpretation of HAADF images, enabling shape and thickness data to be understood.

\section{Introduction}

Secondary electrons (SEs) are produced when an electron beam interacts with matter. This signal is widely used in the scanning electron microscope (SEM). The peak intensity of the SE energy distribution occurs at about $2 \mathrm{eV}$, and such electrons escape from within a few nanometers of the surface. Thus, the SE signal is highly surface-specific, and the image resolution is a function of the imaging probe [1]. Typical field emission gun (FEG) SEMs have imaging resolutions of just a few $\mathrm{nm}$ in SE mode. The great strength of SE imaging is in providing high-resolution surface (topographic) information.

Scanning transmission electron microscopy (STEM) uses a scanning electron probe, much like in SEM. However, the STEM signals originate from electrons transmitted through a thin specimen. Conventional STEM imaging of crystalline specimens is based on brightfield (BF) (Bragg diffraction contrast) and high-angle annular dark-field (HAADF) signals. These provide crystallographic and mass-thickness information, respectively. The resulting projection images impart little direct information about the surface morphology. In the case of regularly shaped particles (for example, spheres), the morphology can be inferred from the image contrast. However, for irregularly shaped particles of varying composition, this becomes difficult.

Despite its utility, SE imaging is not available on most conventional STEM instruments. SE detectors were an option on some JEOL STEMs of the 1980s/90s, such as the JEM-2000FX. At that time, this type of STEM-based SE imaging surpassed the capabilities of some of the best $\left(\mathrm{LaB}_{6}\right)$ SEMs of the day [2].

SE detectors have been installed on a small number of dedicated STEMs and have achieved sub-nm resolution [3-5]. When combined with aberration correction, atomic-resolution SE imaging has been demonstrated [6, 7]. However, for atomic-scale imaging, HAADF produces higher contrast and intensity and has a much lower sensitivity to surface preparation than SE mode. The major advantage of STEM SE imaging is as a complementary technique to HAADF, typically at magnifications well below those necessary for atomic resolution.
The JEOL company has recently released a novel multichannel plate analyzer (BEI) detector option for their aberration-corrected JEM-ARM200F platform, which enables efficient imaging with secondary electrons (SEs) and backscattered electrons (BSEs). STEM BF and HAADF signals may be acquired concurrently with SE or BSE signals, enabling conventional STEM images to be correlated with SEM-like information. This article describes some practical applications of STEM-based SE and BSE imaging for a range of materials.

\section{Materials and Methods}

Various specimens (on carbon films) were imaged: sputtered gold to demonstrate atomic resolution, amorphous boron nitride spheres to show depth effects, mineralized biochar and porous titanium dioxide to reveal surface details, sulfur nanoparticles on graphite to highlight contrast and resolution advantages, and tantalum oxide to show control over depth of field. Particulate specimens were prepared by either light grinding or ultrasonic agitation of the powder in ethanol, then placing a drop of the suspension onto a carbon support film. No subsequent coating of these specimens was performed.
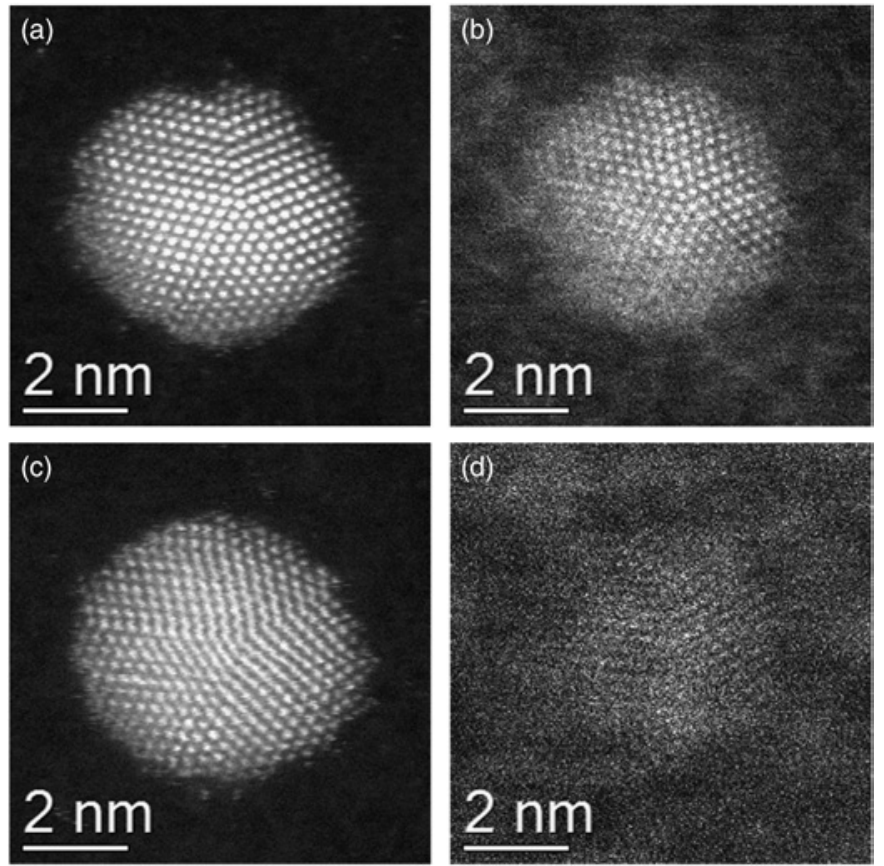

Figure 1: Image sequence of a twinned gold particle on carbon. Under continuous beam scanning, atomic-resolution HAADF and SE images were acquired simultaneously: (a) HAADF at $0 \mathrm{~s}$, (b) SE at $0 \mathrm{~s}$, (c) HAADF at $120 \mathrm{~s}$, and (d) SE at $80 \mathrm{~s}$ electron beam exposure. 


\section{Incomparable...}

\section{...and still innovating}

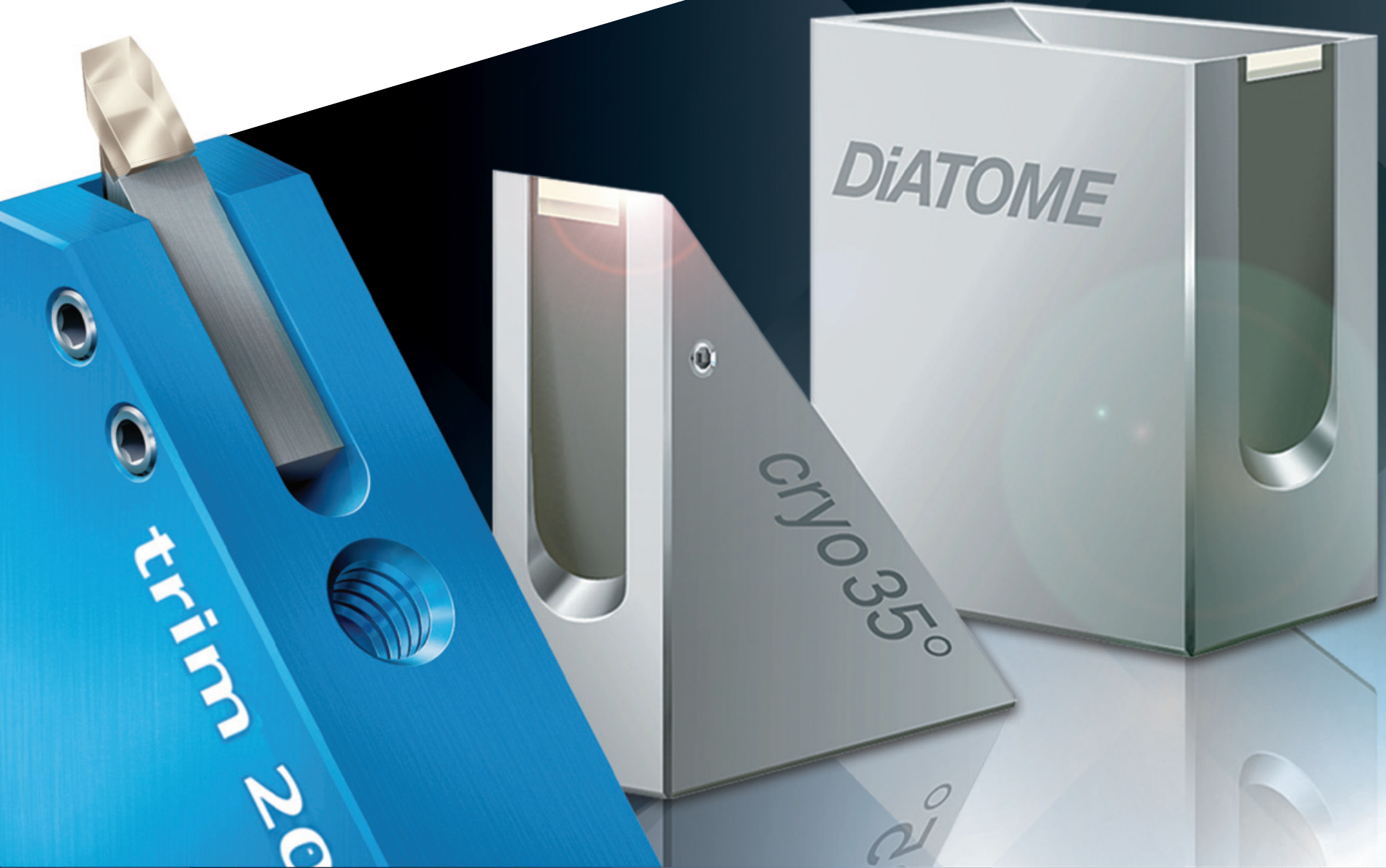

\section{DIATOME diamond knives}

ultra $45^{\circ} \cdot$ cryo $\cdot$ histo ultra $35^{\circ} \cdot$ histo jumbo cryo immuno • ultra sonic ultra AFM \& cryo AFM trimtool 20 - trimtool 45 trimtool 90

Over 40 years of development, manufacturing, and customer service

\section{DIATOME U.S.}

P.0. Box $550 \bullet 1560$ Industry Rd. • Hatfield, Pa 19440 Tel: (215) 412-8390 • Fax: (215) 412-8450

email: sgkcck@aol.com •www.emsdiasum.com

\section{Free customer service}

Sectioning tests with biological and material research specimens of all kinds. We send you the sections along with the surfaced sample, a report on the results obtained and a recommendation of a suitable knife. Complete discretion when working with proprietary samples.

Re-sharpening and reworking service

A re-sharpened Diatome diamond knife demonstrates the same high quality as a new knife. Even knives purchased in previous years can continue to be re-sharpened. The knives can be reworked into another type of knife for no extra charge, e.g. ultra to cryo or $45^{\circ}$ to $35^{\circ}$

\section{Exchange service}

Whenever you exchange a knife we offer you a new DiATOME knife at an advantageous price. 

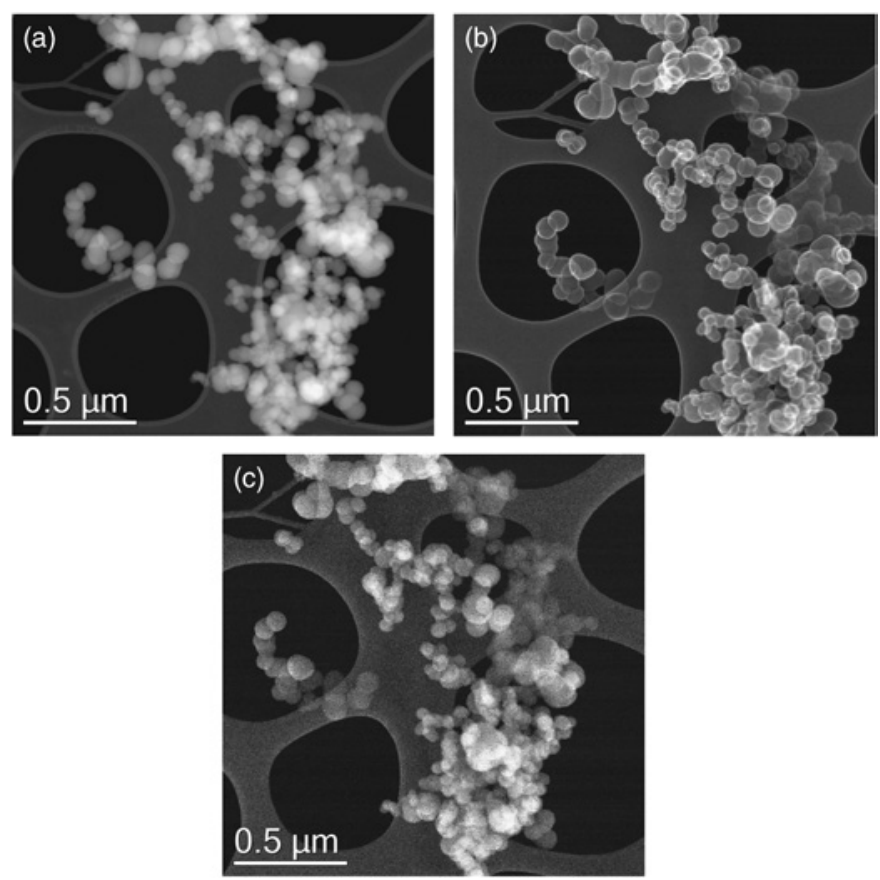

Figure 2: Amorphous boron nitirde spheres on lacey carbon. (a) HAADF image showing no depth information. (b) SE image and (c) BSE image. Both of the latter images highlight which particle clusters lie above, and which lie below, the carbon support film.

Specimens were examined in a JEOL JEM-ARM200F operating at $200 \mathrm{kV}$. This was fitted with a JEOL BEI detector operated in SE and BSE topographic modes. This imaging mode is set in software that controls detector biasing. The only user adjustment is brightness and contrast.

Analytical conditions were used for imaging: $40 \mu \mathrm{m}$ condenser aperture and $0.1 \mathrm{~nm}$ probe size ( $155 \mathrm{pA}$ ). Images were acquired using Gatan's DigiScan hardware and DigitalMicrograph software. No subsequent image filtering or processing was carried out.

\section{Results}

Atomic resolution SE imaging. Figure 1 shows an image sequence obtained from a gold particle on a carbon film. The HAADF image (Figure 1a) shows the multiply twinned gold lattice. A fast Fourier transform (FFT) analysis (not shown) indicated an image resolution of $0.102 \mathrm{~nm}$. The SE
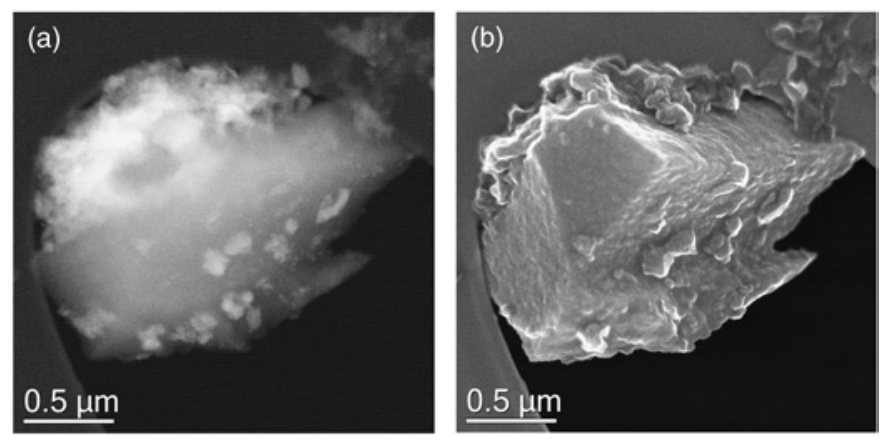

Figure 3: Mineralized biochar fragment. (a) HAADF image and (b) SE image of the same particle. Fe-rich mineral particles decorate the surface of the slab-shaped biochar particle. image taken concurrently with the HAADF image (Figure 1b) also shows lattice resolution $(0.123 \mathrm{~nm})$. The HAADF and SE images of this region were acquired continuously using $20 \mathrm{~s}$ acquisition times. After $80 \mathrm{~s}$ of continuous scanning, the SE lattice contrast had faded considerably (Figure 1d); whereas, the HAADF image acquired after $120 \mathrm{~s}$ of scanning still showed strong lattice contrast (Figure 1c). Minor shape changes and tilting of the crystal occurred due to irradiation effects, and a minor loss of HAADF contrast was observed. The significant degradation of the SE signal is not due to tilting, since the HAADF signal is more sensitive to orientation than the SE signal [6]. A build-up of a thin layer of carbon contamination during the continuous bombardment by the electron beam degraded the SE signal from the gold. The low energy SEs (about $2 \mathrm{eV}$ ) are readily absorbed by carbon layers of just a few $\mathrm{nm}$ in thickness [6]. In contrast, the high-energy $(200 \mathrm{keV})$ HAADF signal is much less affected by such layers.

The atomic-resolution SE imaging shown is an order of magnitude better than that which can be routinely achieved with typical FEG SEM instruments. However, the HAADF image is generally much superior for atomic-resolution imaging. Thus, the primary benefit of SE imaging is realized at lower magnifications where morphology, rather than atomic structure, is of interest.

Depth information and BSE imaging. Figure 2 shows HAADF, SE, and BSE images of amorphous boron nitride spheres. The HAADF image (Figure 2a) is a projection image and thus contains little depth information. In contrast, both the SE and BSE images show which particle clusters lie above and which lie below the carbon film. For X-ray microanalysis, particles projecting over the holes in the film are preferred. However, usually nanoparticulates reside on the support film leaving the holes unpopulated. It is preferable to analyze particles that sit on top of the support film to avoid absorption effects, especially for light element analysis.

The BSE image (Figure 2c) has a much lower signalto-noise ratio than that of the corresponding SE image (Figure 2b). The BSE signal yield increases with atomic number $(Z)$. The very low $Z$ elements of boron, carbon, and nitrogen in this specimen therefore represent a worst case for imaging with this mode.

Thickness and phase disposition information. Figure 3 shows a biochar particle (pyrolysed organic material) following soil exposure. Soil chemistry and microbial activity cause a range of mineral phases to precipitate on the biochar. Understanding the chemistry and mineralogy of this process helps in understanding how biochars improve soil fertility and water retention [8].

The HAADF contrast of the biochar (Figure $3 a$ ) is complex because of the irregular shape and compositionally diverse components; the contrast being a function of both $Z$ and thickness. This makes interpretation of the projection image difficult. Here, the SE image (Figure 3b) has a transformational effect for understanding the material. The particle shape and orientation clarify the contrast variations related to thickness. The small bright regions in the HAADF image are associated with iron-rich particulates. 


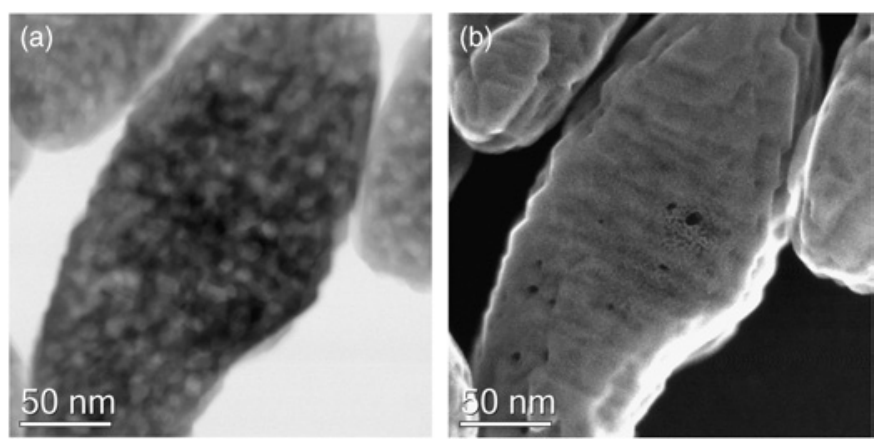

Figure 4: Highly porous $\mathrm{TiO}_{2}$ catalyst support imaged in (a) STEM brightfield and (b) SE image mode. Despite the high degree of internal porosity, very few pores penetrate the surface.

Internal versus external structure. Highly porous oxides are ideal supports for catalysts. The interior of the $\mathrm{TiO}_{2}$ crystal in Figure $4 \mathrm{a}$ is extremely porous. However, this $\mathrm{BF}$ image provides little topographic information. The SE image (Figure $4 \mathrm{~b}$ ) shows that only a limited number of pores extend to the surface, and this may act as a bottleneck to gas exchange. The mottling on the crystal surface is due to small steps or terraces. Such features appear bright since SE emission can occur from the upper surface and exposed side simultaneously [1].

Contrast-limited resolution. Figure 5 shows a dispersion of crystalline sulfur on graphitic carbon. The HAADF image (Figure 5a) shows good contrast between the larger sulfur particles and the thinner regions of graphitic carbon. The smallest sulfur particle that can be resolved is around $6 \mathrm{~nm}$. This limit of particle detection is controlled not by the probe diameter $(0.1 \mathrm{~nm})$, but by contrast limitations. Sub- $6 \mathrm{~nm}$ sulfur particles located on thick regions of carbon produced insufficient contrast to be visible. Superposition of particles on the upper and lower surface of the carbon also degrade the contrast. The SE image (Figure 5b) reveals only particles on the upper surface, detecting crystals as small as $3 \mathrm{~nm}$. The SE contrast between the particles and carbon is largely a function of topography, with the carbon support thickness having little effect on the resolution and contrast [6]. For particle size determination, the SE image is superior to the HAADF.

Depth of field. Aberration-corrected STEM probes typically have a high convergence semi-angle. High image resolution is obtained at the expense of depth of field.
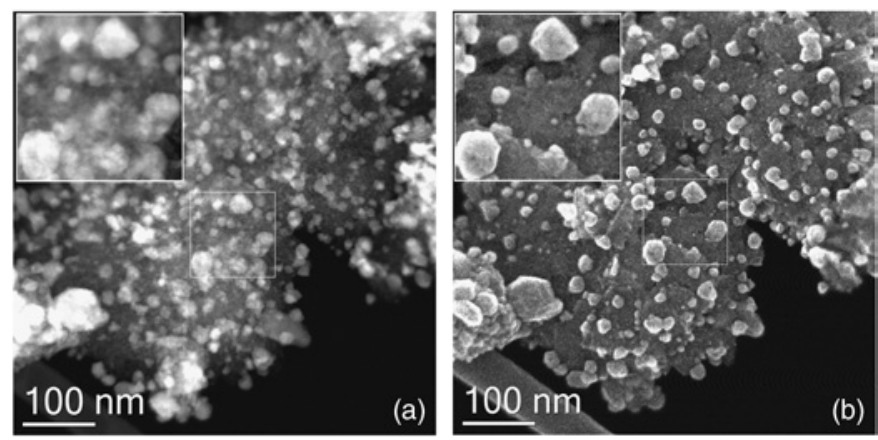

Figure 5: Sulphur nanoparticles dispersed on graphite: (a) HAADF image and (b) SE image. Inset (top left) shows details of the same region in both images. Contrast and resolution are superior in the SE image. Width of inset box $=120 \mathrm{~nm}$.
The depth of field $\left(\Delta_{\text {eff }}\right)$ can be estimated using Equation 1 [9], where $\lambda$ is the electron wavelength $\left(2.51 \times 10^{-12} \mathrm{~m}\right), \alpha$ is the beam convergence semi-angle, $\mathrm{C}_{\mathrm{c}}$ is the chromatic aberration coefficient $\left(1.4 \times 10^{-3} \mathrm{~m}\right), \Delta \mathrm{E}$ is the energy spread of the electron source (about $0.3 \mathrm{eV}$ for a cold FEG), and $\mathrm{E}_{\mathrm{o}}$ is the beam energy $(200 \mathrm{keV})$.

$$
\Delta_{e f f} \approx \sqrt{\left(\frac{\lambda}{\alpha^{2}}\right)+\left(\frac{\mathrm{C}_{\mathrm{c}} \Delta \mathrm{E}}{\mathrm{E}_{0}}\right)^{2}}
$$

Improved depth of field can be achieved by using a smaller condenser aperture. For example, an aperture diameter of $40 \mu \mathrm{m}$ makes $\alpha=24.9 \mathrm{mrad}$ and yields $\Delta_{\text {eff }}=6.1 \mathrm{~nm}$; whereas, an aperture of $10 \mu \mathrm{m}$ makes $\alpha=6.2 \mathrm{mrad}$ yielding $\Delta_{\text {eff }}=67 \mathrm{~nm}$.

SE images of tantalum oxide obtained with condenser apertures of $40 \mu \mathrm{m}$ and $10 \mu \mathrm{m}$ are shown in Figure $6 \mathrm{a}$ and $6 \mathrm{~b}$, respectively. A $10 \mu \mathrm{m}$ condenser aperture improves the depth of field by a factor of 10 compared with the $40 \mu \mathrm{m}$ aperture. However, the major downside is that it produces a 16 -fold reduction in the probe current. To partially compensate for this, a 3 -fold increase in acquisition time was used. Nevertheless, although not easily visible in Figure 6, the smaller aperture will cause a lower signal-to-noise and a slightly poorer image resolution.

\section{Discussion}

The introduction of a BEI detector to the JEOL ARM200F platform greatly extends its imaging capabilities. The detector is an in-lens type, located above the upper objective lens polepiece. This geometry ensures efficient signal collection [10]. In SEM instruments, on-axis, in-lens detectors have been shown to produce improved contrast and topographic information compared with conventional off-axis Everhart-Thornley detectors [11].

In BSE (topographic) mode, the detector provides morphological information for $\mathrm{BN}$ spheres on a carbon film. However, when compared with the HAADF signal, the contrast and signalto-noise ratio are lower. Because of the high energy $(200 \mathrm{keV})$ of the primary electron beam, contrast is not entirely topographic in either BSE or SE mode. Overlapping particles and those particles located on the underside of the support film show weak contrast. This is to be expected for the high-energy BSE signal. However, the BSE signal can also generate SE signals as it exits the support film's upper surface. In the case of SE imaging on non-continuous support films, low-energy SEs from particles on the underside of the film can also pass upward through holes. Such SE signals are brightest where particle edges are close to a hole in the support (for example, lower left in Figure 2b).

Atomic-resolution SE imaging is readily achieved. More importantly, at lower magnifications the topographic information provides insights into specimen location with respect to support films, which is of use in X-ray microanalysis. It also greatly aids interpretation of the complex contrast in HAADF images that can arise in topographically and compositionally irregular materials. For highly porous materials, the correlation of the internal structure ( $\mathrm{BF}$ imaging) with the surface morphology (SE imaging) provides a more complete understanding of the material. For nanoparticulate materials distributed onto the surface of thick supports, the smallest particles may not be resolved in HAADF images because of insufficient contrast. Because the SE contrast is largely 

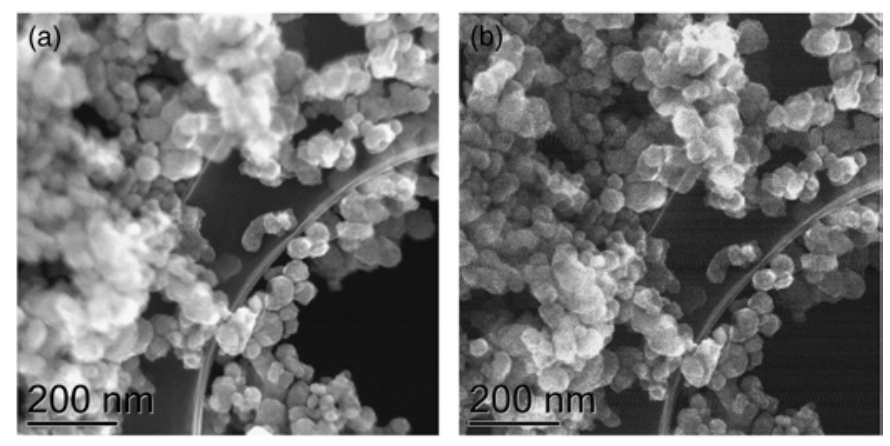

Figure 6: SE images of tantalum oxide powder showing the effect of condenser aperture size on depth of field and signal-to-noise ratio: (a) $40 \mu \mathrm{m}$ and (b) $10 \mu \mathrm{m}$.

independent of the support thickness, smaller particles may be detected in such regions. Because only the upper surface of the specimen is imaged, feature superposition found in projection images is eliminated. The highly converged probe used in aberration-corrected STEM results in a small depth of field. Use of the smallest condenser aperture helps mitigate this problem, albeit at the expense of signal intensity and resolution.

\section{Conclusions}

The addition of a SE/BSE detector to the JEM-ARM200F platform greatly enhances its imaging capabilities. SE images at up to atomic resolution can be readily obtained. However, the real benefit is found at lower magnifications where topographic information greatly aids interpretation of conventional STEM projection images. Correlation of STEM images with SEM data from a separate instrument is no longer necessary, as the microscope described here enables simultaneous acquisition of both.

\section{Acknowledgements}

This research used equipment funded by the Australian Research Council (ARC) with a Linkage, Infrastructure, Equipment and Facilities (LIEF) grant (LE120100104) located at the UOW Electron Microscopy Centre. S. Joseph and M. S. Hossain of the University of Wollongong are thanked for providing specimens.

\section{References}

[1] JI Goldstein et al., Scanning Electron Microscopy and X-Ray Microanalysis, 3rd ed., Springer, New York, 2003.

[2] DRG Mitchell et al., Cement Concrete Res 28 (1998) 1571-84.

[3] RM Allen, Ultramicroscopy 10 (1982) 237-45.

[4] GG Hembree et al., Ultramicroscopy 31 (1989) 111-15.

[5] J Liu and JM Cowley, Ultramicroscopy 37 (1991) 50-71.

[6] H Inada et al., Ultramicroscopy 111 (2011) 865-76.

[7] Y Zhu et al., Nature Materials 8 (2009) 808-12.

[8] J Lehmann et al., Soil Biol Biochem 43 (2011) 1812-36.

[9] R Erni, Aberration-corrected Imaging in Transmission Electron Microscopy: An Introduction, Imperial College Press/World Scientific Pub. Co., London, 2010, p. 304.

[10] BJ Griffin, Scanning 33 (2011) 162-73.

[11] W Kuo et al., Platinum Metals Review 58 (2014) 106-10.

\section{Corion Optical Filters}

\section{Unique Capabilities - Superior Solutions \\ - Hard-coated/Single-element \\ Brilliant fluorescence imaging}

-Hard-coated/Laminated-multi-element Highest signal-to-noise ratios

\section{- Rapid Prototyping \\ Custom Design/Manufacturing}

- High index contrast

Best value

Visit us at www.newport.com

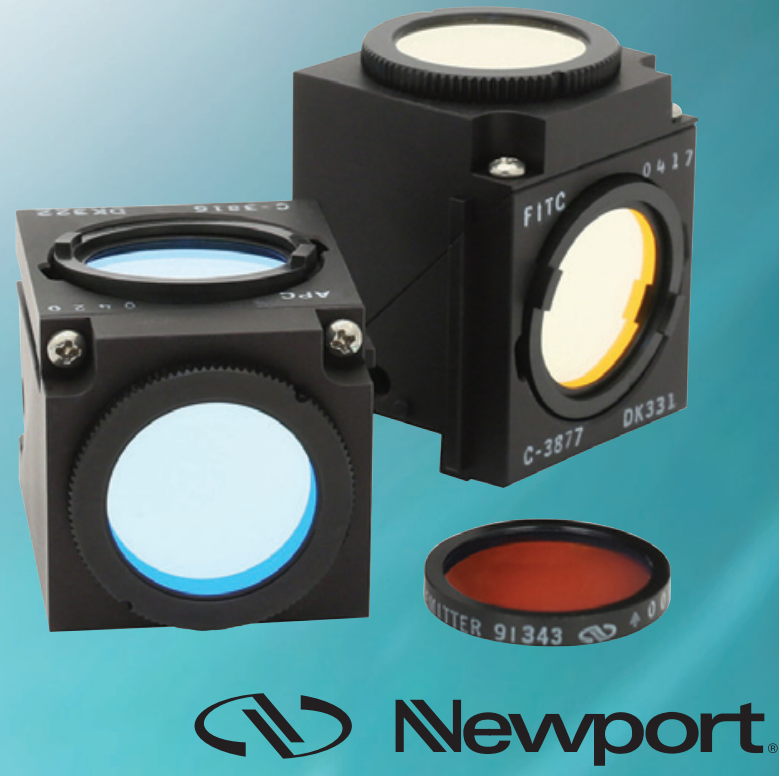




\section{ATMOSPHERE}

Environmental Capability In Any Microscope

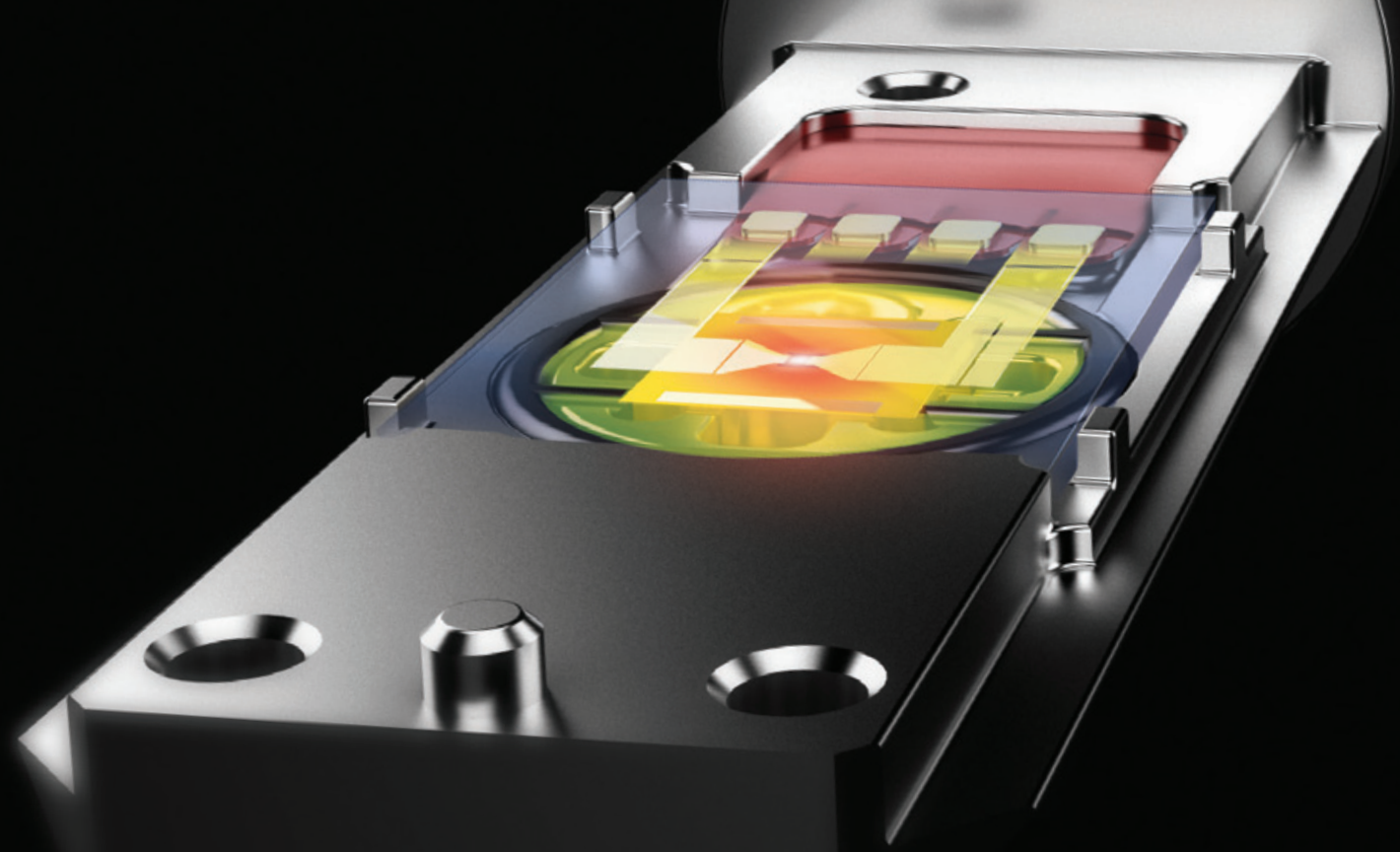

(-8) 1 ATM

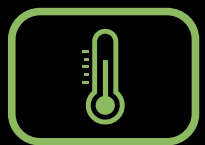

$1000^{\circ} \mathrm{C}$

Maximum Temperature

\|\| Elemental Analysis
Register for the free webinar today via protochips.com

Witness Atmosphere's impressive capabilities by attending Chemistry in the TEM - New Horizons for Catalyst Research, a new webinar provided by Materials Today. 